\title{
SELECTING CONTENTS FOR A NEW VIRTUALIZATION COURSE IN INFORMATION TECHNOLOGY (IT) TRACK
}

\author{
Azad Ali, Indiana University of Pennsylvania, azad.ali@iup.edu
}

\begin{abstract}
The purpose of this study is to research for contents for a newly developed course in computer technology virtualization. The course is being proposed in a new Information Technology (IT) track at a department of Management Information Systems (MIS). IT programs face more frequent changes in their curriculum than other programs. Some IT programs may follow familiar steps and develop new courses that are typical of IT courses (like a course in programming, database, systems analysis or other similar). However, a course in computer virtualization is atypical to other IT courses. Thus selecting contents for a course like this creates unique challenges that need to be addressed differently than those dealt with when introducing typical IT courses.

This paper reviews literature on computer technology virtualization. The goal of this study is to select contents for inclusion in a new course syllabus in computer technology virtualization. Lately, our department has decided to add a new course in computer technology virtualization into our curriculum. Hence we started our research for topics for the course here. We intend to include the course contents we find from our research in this study into the syllabus for our new course.
\end{abstract}

Keywords: Virtualization, Computer virtualization, Hardware Virtualization, Software Virtualization

\section{INTRODUCTION}

Information technology (IT) programs often face difficulties with the selection of contents for new developed courses. This is largely true due to the rapid advances in computer technology which in turn reflects on the curriculum development process (Ali \& Shubra, 2010, Markoff, 2009, Zeweben, 2011). The difficulty with selecting contents for non-common courses may increase due to the lack of familiarity with the contents that need be included.

Recently, the department of Information Systems and Decision Science (ISDS) at Indiana University of Pennsylvania (IUP) has taken steps to introduce a track in IT and began to develop courses for inclusion in the IT track. One of the courses that were selected for the new IT track is a course in computer technology virtualization. However, in order to include the course in the track (and hence into the department curriculum), our department has to prepare course syllabus that include course description, course objectives and course contents. We determined that the best way to start working on the virtualization syllabus is to begin searching for contents for the course. So our task in this paper is to research for and select contents for our proposed virtualization course. The contents need to be relevant to the topic of the course. We also have other guiding criteria that we apply when selecting contents for all of our courses. These issues are addressed in this paper as well. The remainder of this paper is divided into the following sections:

- This paper first reviews literature regarding the topic of virtualization to explain different vocabulary, brief history and their inclusion academic curriculum.

- Then the paper discusses the common virtualization platforms including hardware, software and other types of virtualization.

- The next section explains the other institutional considerations that are typically taken into consideration when adding a course to the curriculum.

- Last the paper elaborates at the experience of the ISDS and suggests contents for their course. A recommendation for course contents is included at the end of the paper. 


\section{VIRTUALIZATION - LITERATURE REVIEW}

Although the topic of computer technology virtualization has been in existence for some time but their introduction into academic courses is more recent. So before delving into the details of course selection, we present a few ideas about virtualization so to gain a basic understanding of this topic and then to be able to make more informed decision about the appropriate contents for our proposed course.

\section{Virtualization - Basic Meaning}

In seeking to understand the meaning of the word "virtualization", we tried to find a definition of the word in the dictionary in our common English language. We searched the word in Webster's dictionary and then in Dictionary.Com but did not find a definition of the word. Instead, we found a definition of the word "Virtual" and is defined as "Very close to being something without being it" and also "as being such in power, force, or effect, though not actually or expressly". From these two definitions, we understand the word to mean virtual something is not the real something but it represents and has similar characters of the real something.

The understanding of the word "virtual" was transferred into the computer technology and extended further into terms, functionalities and features of the technology. Hess and Newman (2010) defined virtualization as "Physical abstraction of computing resources". This means in that some computing resources (like software application, CPU power, other hardware resources) that reside on one computer are also shared or used by other computers or other people.

Hammersley (2006) echoed the earlier definition and described virtualization as "abstraction of resources" (P. 2). Vouk (2008) provided a more comprehensive description and explained that virtualization "allows abstraction and isolation of lower level functionalities and underlying hardware. This enables portability of higher level functions and sharing and/or aggregation of the physical resources" (P. 237).

Based on reviewing the above definitions, the basic meaning that applies to virtualization there are two (or more) computers, one that has resources (functions) and the other computer(s) extract (use) resources from the first computer. Extending this meaning further reveals that through virtualization, many computers with limited resources connect and take properties from the computer with the additional resources.

\section{Virtualization - Brief History}

Virtualization as a computer technology topic and their inclusion into academic courses started at different times and went through different stages to get to the point it is being used now. Miller and Pegah (2007) noted that "Virtualization has a long history, starting in the mainframe environment in the 1960's and arising from the need to provide isolation between users" (P. 257). Crosby (2006) explained that the start of virtualization goes back to the dates of "Time Sharing" computers when computing recourses were scarce and multiple users used to be connected to share a single expensive computing resources. Crosby still refer to the 1960s and 1970s that saw the major development of virtualization and noted that a major milestone regarding virtualization happened with the development of IBM System/360 in 1972.

One of the premiere time-sharing systems is the VM/370 Time-Sharing system. This is significant in the development of virtualization, Creasy (1981) noted the following about the significance of this system in virtualization

$\mathrm{VM} / 370$ is an operating system which provides its multiple users with seemingly separate and independent IBM System/370 computing systems. These virtual machines are simulated using IBM System/370 hardware and have its same architecture. In addition, VM/370 provides a singleuser interactive system for personal computing and a computer network system for information interchange among interconnected machines. VM/370 evolved from an experimental operating system designed and built over fifteen years ago. (p. 483). 
A couple points can be highlighted from the statement above: first, the IBM System/370 provides single interactive system for personal computing and network access at the same time. This is significant knowing that at the time this system was developed such single interactive system did not exist. Second, the fifteen years mentioned in the quote. The fifteen years mentioned in the quote refer to the years before publishing the article in 1981, this shows that the grassroots of virtualization started years before and that IBM System/370 contributed to their development in the shape they are now.

On a different note and way before virtualization spiraled to increase their role in computing, the movie titled "Virtual Reality" was first shown in theaters in 1995 (Craig, Sherman and Will, 2009). Although the film is a fiction like resemblance of real life but it highlighted that through technology, a virtual something (like virtual world) can be created that mimics or exceeds the original thing that was created after.

Aside from the developments described above, further major developments in virtualization have been associated with the introduction of a platform or hardware systems. In other words, the development of a certain hardware/software that is strongly connected to virtualization made milestones in the timeline or history of virtualization development. Hess and Newman (2010) listed some of these platforms:

- VMware Server

- VMware ESXi

- Critix XenServer

- $\quad$ Microsoft Virtual PC

- $\quad$ Microsoft Hyper-V

- $\quad$ Sun Microsystem Virtual Box

The most recent milestones in virtualization focused on the development of major platforms. In 1999 for example, VMware introduced the first x86 virtualization product (Adams and Agesen, 2006). This is a major milestone because it increased the role of VMWare in the virtualization market. VMware extended their role further in the market of virtualization when they decided to provide high technology virtualization to everyone for free. This led to simplifying desktop virtualization (Halter and Wolf, 2005) which in turn further extended the use of virtualization to various technologies.

The most recent technological developments have witnessed substantial increase in the use of virtualization technology notably in the gaming area but it also extended into other areas as well. Seetharman and Hirschauge (2015) predicted the increase use of virtualization on Facebook where viewers can view a 360 degree of a video and it allows visitors to change their viewing perspectives by tilting their phones. Mims (2015) described that virtual reality is not just about games anymore, instead Mims predicted that virtual reality "offers strong potential for performing 'mundane tasks' (page B1) as well. Based on the events listed here, one can predict that virtualization technology is here to stay and that in most likelihood it will expand to cover other wider areas as well.

\section{Virtualization - Basic Concepts}

Similar to any other field of study, virtualization has its own concepts and basic terms that need to be understood before delving deeper into the subject. We decided to identify the basic terms used in virtualization and then to explain them further so to help us in selecting for our proposed course. Upon review of the basic concepts, we decided to explain about the following terms regarding virtualization:

\footnotetext{
- Host machine

- Guest machine

- Hypervisor

- Snapshot

- Migration

- Emulation
} 


\section{Issues in Information Systems \\ Volume 17, Issue I, pp. 47-57, 2016}

A host machine is the machine with the resources that can be shared by other computers or platforms. Since this machine has the resources, it offers the resources to other machines. A guest machine is the machine that is using the resources offered by the first machine (Liebowitz and Fontana, 2013). The word guest is used to denote that it resides inside the first machine or its use of the resources depends on the first machine - thus used the word guest.

The communication (or traffic lines) between the host machine and the guest machines follows procedures, protocols, standards and devices that need to be managed. All the traffic between the host and guest machines is managed through what is termed as hypervisor (Hess and Neman, 2010).

Snapshot is the state of the virtual machine, and generally its storage devices, at an exact point in time. A snapshot enables the virtual machine's state at the time of the snapshot to be restored later. An analogy can be drawn to setting the system restore point in the desktop environment. So when a computer malfunctions, restoring it to earlier point may solve the problem. Similarly, restoring to a snapshot point take the guest machine to the earlier point that the snapshot was taken (Hess and Neman, 2010).

The snapshots described above can be moved to another host machine with its own hypervisor; when the virtual machine is temporarily stopped, snapshotted, moved, and then resumed on the new host, this is known as migration. If the older snapshots are kept in sync regularly, this operation can be quite fast, and allow the virtual machine to provide uninterrupted service while its prior physical host is, for example, taken down for physical maintenance (Dong et al., 2009).

According to Hess and Newman (2010) Emulation refers to the capability to mimic a particular type of hardware for an operating system regardless of the underlying host operating system. In others words, the host hardware works the same way that the original hardware worked, thus the user does not see the difference when working with a virtualized software versus the original working environment (Crosby, 2006).

\section{Virtualization Benefits and Challenges}

According to Halter and Wolf (2005), "Virtualization offers several benefits, including consolidation of infrastructure, ease of replication, normalization of systems and isolation of resources (P. 1)". Baev, Weaver and Weaver (2011) explained that virtualization solution is ideal for organization that face budgetary restrictions and that through virtualization, organizations can find solutions for balancing among users. The solution noted by Baev, Weaver and Weaver study include scalability, system maintenance and efficiency of in terms of cost minimization. Burdonov, Kosachev and Iaknevko (2009) suggested that through virtualization, common sensitive data are more protected in comparison to legacy software that are normally used in some applications.

Libeowitz and Fontana (2013) explained there are benefits that can be gained from business virtualization (that is virtualizing business applications) and noted the following as among the benefits that can be gained:

- High availability

- Disaster recovery

- Scalability

- Performance testing

Hess and Newman (2010) clarified that replacing aging infrastructure is a prime reason that leads organizations to introduce virtualization. They noted further that "Virtualization is a clear winner in the aging infrastructure dilemma (P. 5)". They added that additional benefits from virtualization may include minimizing hardware costs, providing disaster recovery and other benefits that can be sought in terms of software tests.

Although benefits of virtualization are numerous, there are still challenges that face businesses that attempt to virtualize their technologies. Libeowitz and Fontana (2014) for example listed some of the challenges that face the virtualization of business application that includes, supportability, management, security risks and others. 


\section{Issues in Information Systems \\ Volume 17, Issue I, pp. 47-57, 2016}

Laaden and Nieh (2010) explained about some of challenges for virtualization that includes increasing the complexity of operating and maintaining a virtual system, In other words, a virtualized system requires additional and more complex steps to operate and manage that comparable systems.

Pearce, Zeadally and Hunt (2013) were particular in mentioning the risks of security threats that emerge from virtualization. This risks stems from multiple platforms operating on one (host) platform which make it more subject to vulnerabilities than when working on a single system operating.

\section{Virtualization in Academic Curricula}

In our attempt to find content for our virtualization course, we searched for college courses that exclusively teach virtualization. We did not find college courses that are entirely designed to teach virtualization. Instead, we found topics of virtualization that are covered with other course. Thus we decided to go a different path and research in the standard curriculum to see the extent at which virtualization is introduced.

There are a number of organizations that work to develop standards for various technology fields: The Association for Computing Machinery (ACM), the Institute of Electrical and Electronics Engineers (IEEE), the Association of Information Systems (AIS) and the Association of Information Technology Professionals (AITP). These organizations have worked at many levels to develop standard curriculum for different computer disciplines. They have developed a number of documents in this regard and have indicated that they will continue to do so to develop additional documents as the need for developing such documents arises. The initial focus of these efforts was to develop a standard curriculum for the computer science majors. This evolved into a larger number of standard curricula to cover wider range of computer related (or IT) programs.

The documents that are developed by these organizations are labeled as computing curriculum, though not officially considered "standards"; they have been widely used in curriculum development and accreditation in this field. In fact, due to their wide use in higher education, these documents are considered as de facto standard curriculum in the information technology fields.

For the purpose of this paper, we searched the most recent standard curriculum. We selected to search in this study:

- Information Technology 2008, Curriculum Guidelines for Undergraduate Degree Programs in Information Technology (Or IT2008)

- IS 2010: Curriculum guidelines for undergraduate degree programs in information systems (or IS2010)

- Computer Science Curricula 2013 Curriculum Guidelines for Undergraduate Degree Programs in Computer Science (or CS2013)

In IT2008, we found that virtualization is mentioned within the topics that covered for operating system on page 106 in core learning outcome and advances learning outcomes. In IS2010, the topic of virtualization is mentioned in IS2010.3, Enterprise Architecture on page 53. Virtualization of storage and systems and also in IS 2010.4 IT Infrastructure on page 54. In CS2013, virtualization is mentioned as part of the IAS (Information assurance and security) on pages 102-106, within operating systems (page 138 - 142) and in PD/Cloud computing on page 156 and then in SD systems fundamentals.

\section{VIRTUALIZATION PLATFORMS \& TOPICS}

Hess and Newman (2009) explained that there are different platforms for virtualization including application, network and storage platforms. Halter and Wolf (2005) on the other hand listed several types of virtualizations that included hardware emulators, application virtual machines, mainframe virtual machine, operating system virtual machine and parallel virtual machines - all within the topic of virtualization. 
This kind of a mix in virtualization platforms needs special attention when selecting topics for a course. Such attention helps in the selection of the specific platforms that are more applicable and more helpful to cover in the course. This section explains about the various virtualization platforms and topics that were reviewed for this study to enable us to select the appropriate topics for our suggested course.

\section{Hardware Virtualization}

Hardware virtualization is the most common type of virtualization and it is the type of virtualization that is considered a default if no type is mentioned when discussing and explaining about virtualization (Hess and Newman, 2009). Laaden and Nieh (2010) on the other hands talked about the techniques of hardware virtualization and explained that they (hardware virtualization) aim to separate underlying hardware architecture from the operating system so that "an entire OS environment and associated applications can be executed in a virtual environment" (P. 17).

\section{Software Virtualization}

This is also called application virtualization. Kim and Hariri (2012) introduced the following type of services that are taken into account when applying application virtualization:

- Application control services

- Application data services

- Application editing services

- Application management services

- Application virtualization services

\section{Operating System Virtualization}

Crosby (2007) explained that an operating system virtualization environment "provides each virtualized operating system (or guest) the illusion that it has exclusive access to the underlying hardware platform in which it runs" (P. 34). This technology enhancement made possible that multiple operating systems be able to work on the same machine (an operating system working inside another operating system). All operating systems then share the resources on a single hardware server.

The virtual environment inside this machine can be configured to host multiple operating systems (they are called virtual operating systems). The virtual environment can be set up so that the users of the virtual operating systems work as if they are working on a real physical operating system (Smith \& Nair, 2005).

\section{Desktop/Notebook Virtualization}

Desktop virtualization was introduced into academia first as a mean to provide cost effective solution in light of the budgetary restrictions and the attempts of various organization to cut their technology budgets (Baev, Weaver \& Weaver, 2011). Using desktop computers as an option for the purpose of reducing cost continue to be suggested because other alternatives did not provide similar cost savings. However, as the cost of other alternatives (laptops, notebooks) continue to decline, they are also suggested to be included in the virtualization process.

A notebook computer that is aging can be used simply as display and keyboard/mouse device, connecting to a remote session running on a server. Miller and Pegah (2007) listed examples of using notebooks in a virtualized environment that include the following examples:

- Augmenting remote access to specific, high end applications that are aren't always needed,

- Applications that do not run on native operating system, or

- Applications that have performance requirements beyond the capacity of the local system.

- Provide solutions for high end graphics workstations

- Provide data solutions in high end data centers 


\section{I/O Virtualization}

Input/output (I/O) virtualization is a methodology to simplify management, lower costs and improve performance of servers in enterprise environment. Onrago, Cox and Rixner (2008) explained that the challenge in I/O virtualization is having multiple virtual machines residing on a single host machine to share the services and resources available on the host machine. This requires complex scheduling tasks in order to meet the demand of the multiple operating guest operating systems (Dong et al., 2009). The added that this involves scheduling of different events and distinguishing the priority of each event that is handled by the SPU.

\section{Virtualization Security}

Technology security is a topic that is addressed, studied and emphasized repeatedly in different IT fields. Virtualization technology security is no exception, it needs be addressed from the general aspect of IT security but it also need to addressed from the perspectives of hosting machines and the difference of platforms that are embedded in one machine.

In a study conducted by Burdonov, Kosachev, and Iakovenko (2009) and discussed the sensitive issues that need to be dealt with in a virtualized environment especially when sensitive data are shared in the process. The same study suggested that separation of privileges is the best approach to dealing with security issues in a virtualized environment. By separation of privileges, the authors suggested to physically isolating some computers from the network to minimize data leaks and to protect sensitive data.

\section{OTHER INSTITUTIONAL CONSIDERATIONS}

Selecting content for an IT course (or for any other courses in this case) is subject to other considerations. This section explains some of the other factors when we are considering when deciding on contents for our virtualization course. We will address these considerations from two perspectives, first from a point of redundancy and second from the point of marketability of the course.

\section{Reduce Redundancy and Duplicate of Materials}

Reducing redundancy is a common theme that is echoed in various computer technology courses. In database topics, some consider reducing redundancy as a goal that is sought when normalizing the database tables (Pratt \& Adamski, 2002). Reducing redundancy is considered one of the steps that contribute to successful database design for business applications. A similar sentiment is echoed in academia when designing courses. Redundant materials across courses are known to waste time and leads to inconsistencies. Moreover, duplication of course content leads to unfavorable attitude from students and teachers also.

Technology fields are known to have redundant and duplicate materials among courses. A department for example that is teaching two programming courses may repeat concepts about design structure, sequence, selection, and looping concepts in each of the two courses they teach. A course in hardware design may repeat content regarding CPU, hard drives, and motherboard that may be covered in earlier computer literacy course. Redundancy in technology course seems to be inevitable. Nevertheless, educational common sense reveals that introducing a new course that minimize the redundant information is helpful to the students and to the program that offers it as well.

\section{Marketability of Course}

By marketability of the course, we mean here to attract new students into the program (and into the course) once we complete the development of the course and start offering it. The marketability of the course can be derived from the reputability of the subject being discussed. If the topics being covered have future, the common sense is that the course will be marketable and there will be more students taking or enrolling in the course. The opposite is true. 
There are many literatures that predict a bright future for computer technology virtualization. Crosby (2007) for example explained that virtualization appears not just another short-lived technology solutions, instead Crosby predicted that virtualization is poised to deliver profound changes to the way that both enterprise and consumers use computer systems" (P. 34). Ongaro, Cox and Rixner (2008) predicted an increase in interest for computer technology virtualization.

\section{THE CASE}

This section explains about our department (the department of Information Systems and Decision Science) and the development and the process that led first to the thinking of adding a course and second the steps taken to finalize the selection of content for the virtualization course.

\section{Selecting the New Course}

Recently, two departments at IUP have joined together. These two departments are the Department of Management Information System (MIS) and the department of Technology Support and Training (TST). The department of Information Systems and Decision Science (ISDS) at Indiana University of Pennsylvania (IUP) was formed as a result of the merger between the MIS and the TST departments.

Before the merger, the basic understanding was that the faculty in the old TST department will be merging their courses into the new formed department. That is, the TST faculty will develop an Information Technology track within the MIS degree whereby they incorporate some of their old courses. However, as the revision process started, the ISDS department made it clear that they will not accept the old TST courses, instead they suggested to start new courses. Two conditions were set for the development of the new courses: First, the content of the new courses need not to overlap with the content of the department of courses and the other IT department at IUP - the department of computer science. Second, the courses need to be marketable and have enough rigor that it attracts new students and maintain the thoroughness that the department has offered for long in their courses.

\section{Selecting Content for the New Course}

The department was clear about their desire to have a new course in virtualization but did not agree on what is to be taught in the course. Thus, we decided to conduct this research to find applicable content for this course. The department went back and forth over several revisions of the course, but after a while we come to conclusion about what need to be covered in this course. The following section presents a list of suggested content that we are going to include in our proposed course in computer technology virtualization.

\section{RECOMMENDATION}

After numerous attempts, the author has provided the recommendations for content in the virtualization course. The following is an itemized list of the content that we recommended for our course IFMG254 Computer Technology Virtualization.

A. Virtualization overview

1. History of virtualization

2. Basic concepts of virtualization

3. Time sharing systems

4. Distributed computing system

5. Virtual machine anatomy

6. Virtual machine preparation 
7. Virtual machine file system

B. Applying virtualization

1. Virtualization configuration

2. Deployment of virtualization

3. Virtualization and security issues

4. Preparing budget for virtualization

5. Migration to virtualization

6. Virtual Private Network

7. Virtual local area network

C. The virtual environment infrastructure

1. Hardware role in virtualization

2. File Systems and Storage

3. Towers racks and blades

4. Chip vendors in virtualization

5. Cores and sockets in virtualization hardware

6. Virtualization vendor choices

7. Virtualization through cloud computing

D. Operating systems virtualization

1. File systems virtualization

2. Embedded systems virtualization

3. Environmental support for virtualization

4. Virtual operating systems interfaces

5. Abstraction virtualization

6. $\quad \mathrm{I} / \mathrm{O}$ and memory issues in virtualization

7. Virtualization challenges in operating systems

E. Enterprise virtualization

1. Installing virtual machine on enterprise servers

2. Deploying virtual machine on enterprise servers

3. Managing enterprise virtual solutions

4. Virtual machine backup and recovery

5. Virtual machine file systems

6. Virtual machine clusters

7. The virtualized information system

F. Virtualization security

1. Fundamentals of virtualization security

2. Virtualization architecture

3. Vulnerabilities in virtualization

4. Challenges for securing virtualized environment

5. Protecting virtualized environment

6. Effects of virtualization on information security

7. Virtualization and forensics 


\section{REFERENCES}

Adams, K., \& Agesen, O. (2006). A comparison of software and hardware techniques for x 86 virtualization. ACM Sigplan Notices, 41(11), 2-13.

Ali, A., \& Shubra, C. (2010). Efforts to reverse the trend of enrollment decline in computer science programs. The Journal of Issues in Informing Science and Information Technology, 7, 209-225.

Baev, S., Weaver, C., \& Weaver, C. (2011). Cost-efficient desktop vitualization experience at Georgia Southwestern State University. Journal of Computing Sciences in Colleges, 27(2), 188-195.

Burdonov, I., Kosachev, A., \& Iakovenko, P. (2009, March). Virtualization-based separation of privilege: working with sensitive data in untrusted environment. In Proceedings of the 1st EuroSys Workshop on Virtualization Technology for Dependable Systems (pp. 1-6). ACM.

Computer Science Curricula 2013, Curriculum Guidelines for Undergraduate Degree Programs in Computer Science. ACM. Available at: http://www.acm.org/education/CS2013-final-report.pdf

Craig, A. B., Sherman, W. R., \& Will, J. D. (2009). Developing virtual reality applications: Foundations of effective design. Morgan Kaufmann.

Creasy, R. J. (1981). The origin of the VM/370 time-sharing system. IBM Journal of Research and Development, 25(5), 483-490.

Crosby, S., \& Brown, D. (2006). The virtualization reality. Queue, 4(10), 34-41.

Dong, Y., Dai, J., Huang, Z., Guan, H., Tian, K., \& Jiang, Y. (2009, May). Towards high-quality I/O virtualization. In Proceedings of SYSTOR 2009: The Israeli Experimental Systems Conference (p. 12). ACM.

Halter, Chris Wolf Erick M. Halter, C. W. E. M. (2005). Virtualization: From the Desktop to the Enterprise (Books for Professionals by Professionals).

Hammersley, E. (2006). Professional VMware Server. John Wiley \& Sons.

Hess, K., \& Newman, A. (2010). Practical virtualization solutions: virtualization from the trenches. Pearson Education.

IS 2010, Curriculum Guidelines for Undergraduate Degree Programs in Information Systems. ACM. Available at http://www.acm.org/education/curricula/IS\%202010\%20ACM\%20final.pdf

Information Technology 2008, Curriculum Guidelines for Undergraduate Degree Programs in Information Technology. ACM. Available at: http://www.acm.org//education/curricula/IT2008\%20Curriculum.pdf

Kim, D., \& Hariri, S. (2012). Virtual Computing: Concept, Design, and Evaluation (Vol. 633). Springer Science $\&$ Business Media.

Laadan, O., \& Nieh, J. (2010, May). Operating system virtualization: practice and experience. In Proceedings of the 3rd Annual Haifa Experimental Systems Conference (p. 17). ACM.

Liebowitz, M., \& Fontana, A. (2013). Virtualizing Microsoft Business Critical Applications on VMware VSphere. Pearson Education. 
Markoff, J. (2009). Computer science programs make a comeback in enrollment. The New York Times.

Miller, K., \& Pegah, M. (2007, October). Virtualization: virtually at the desktop. In Proceedings of the $35 t h$ annual ACM SIGUCCS Fall Conference (pp. 255-260). ACM.

Mims, Christopher (2015). Virtual Reality Isn’t Just About Games. Wall Street Journal, August 3rd, 2015, p. B5.

Ongaro, D., Cox, A. L., \& Rixner, S. (2008, March). Scheduling I/O in virtual machine monitors. In Proceedings of the Fourth ACM SIGPLAN/SIGOPS International Conference on Virtual Execution Environments (pp. 1-10). ACM.

Pearce, M., Zeadally, S., \& Hunt, R. (2013). Virtualization: Issues, security threats, and solutions. $A C M$ Computing Surveys (CSUR), 45(2), 17.

Pratt, P. J., \& Adamski, J. (2002). Concepts of database management. Boston: Thompson Course Technology Seetharman, Deepa, Hirschauge, Oer (2015). Facebook Plans App for Virtual Reality. Wall Street Journal, September 14, 2015, p. B5.

Smith, J. E., \& Nair, R. (2005). The architecture of virtual machines. Computer, 38(5), 32-38.

Topi, H., Valacich, J. S., Wright, R. T., Kaiser, K., Nunamaker Jr, J. F., Sipior, J. C., \& de Vreede, G. J. (2010). IS 2010: Curriculum guidelines for undergraduate degree programs in information systems. Communications of the Association for Information Systems, 26(1), 18.

Virtual (2015). In Merriam-Webster.com. Retrieved May 10, 2015 from http://www.merriamwebster.com/dictionary/virtual

Vouk, M. (2008). Cloud computing-issues, research and implementations. CIT. Journal of Computing and Information Technology, 16(4), 235-246.

Zweben, Stuart (2011). Computing degree and enrollment trends. Computing Research Association. 